\title{
Dispersion Compensation with Dispersion Compensating Fibers (DCF)
}

\author{
Manpreet Kaur $^{1}$, Himali Sarangal ${ }^{2}$, Parveen Bagga ${ }^{3}$ \\ Student (M.Tech), Department of Electronics and Communication Eng. GNDU Regional Campus Jalandhar, India ${ }^{1,3}$ \\ Asst.Prof, Department of Electronics and Communication Engineering GNDU Regional Campus Jalandhar, India ${ }^{2}$
}

\begin{abstract}
In this paper, dispersion compensating fibers (DCF) are used to compensate the positive dispersion accumulated over the length of the fiber at 10Gbits/sec and $250 \mathrm{~km}$ of optical fiber with $50 \mathrm{~km}$ of DCF. Three schemes (Pre-compensation, post-compensation and symmetrical-compensation) of dispersion compensation with DCF are proposed. The simulated transmission system has been analyzed on the basic of different parameters by using OptiSystem 7.0 simulator. The results of three dispersion compensation methods are compared in terms of four parameter, which are Q-factor, BER, Eye height and threshold value, investigated at the receiver end. Further, it has been observed that the system needs proper matching between EDFA gain and the length of the fiber for the optimum performance.
\end{abstract}

Keywords: Dispersion compensation, Dispersion compensating fiber (DCF), BER, Q-factor, Eye height.

\section{INTRODUCTION}

When optical signals are transmitted over the optical links, different wavelength components of the signals will generally experience different propagation time due to the fact that the transport medium has different effective refractive indices for different wavelengths. In the recent years, with the rapid growth internet business needs, people urgently need more capacity and network systems. So the demand for the transmission capacity and bandwidth are becoming more and more challenging to the carriers and service suppliers. Under this situation, optical fiber is becoming the most favourable delivering media and laying more and more important role in information industry, with its huge bandwidth and excellent transmission performance. Therefore, it is necessary to investigate the transmission characteristics of optical fiber. The main goal of any communication system is to increase the transmission distance. Loss and dispersion are the major factors that affect the fiber optic communication system. The EDFA (Erbium doped fiber amplifier) is the gigantic change that happened in the optical fiber communication systems; the loss is no longer major factor to restrict the fiber optic transmission. Since EDFA works in $1550 \mathrm{~nm}$ wave band, the average Single Mode Fiber (SMF) dispersion value in this wave band is very big, about 15-20ps / (nm.km-1). So, it is easy to see that the dispersion become the major factor that restricts the long distance fiber optic system. [1]

In this paper, three DCF compensation schemes (postcompensation, pre-compensation and symmetrical/mix compensation) are analyzed to compensate dispersion in SMF. Simulation studies show that post compensation scheme is the best. It can greatly reduce the influences of the fiber nonlinearity and increase the transmission distance greatly.

The rest of the paper is organized as followed. In section II, The causes and effects of dispersion on optical transmission system are presented. In section III, The present dispersion compensation technologies. In section
IV, DCF dispersion compensation technique is presented. Section V presents the transmission system simulation with OptiSystem 7.0 and different parameters are analyzed and compared. Section VI concludes the paper.

\section{EFFECTS OF DISPERSION ON OPTICAL COMMUNICATION SYSTEM}

Dispersion characterized optical fiber in terms of maximum transmission speed. When different wavelengths of light pulses are launched into the optical fiber, these pulses travelled with different speeds due to the variation of refractive index with wavelength. The light pulses tend to get spread out in time after travelling some distance in fiber and this is continued throughout the fiber length. This phenomenon of broadening of pulse width is known as dispersion. The pulse width gradually increases and the peak power of pulse reduces. Dispersion of the transmitted optical signal causes the distortion for both digital and analog transmission along optical fibers. Each pulse broadens and overlaps with its neighbours eventually becoming indistinguishable at the receiver input. This effect is known as inter symbol interference. [2]

Dispersion limits the information capacity at high transmission speeds. Dispersion reduces the effective bandwidth and at same time it increases the BER due to an increasing inter symbol interference. In order to remove the spreading of optical pulses, dispersion compensation is required. In single-mode fiber, performance is primarily limited by chromatic dispersion (also called group velocity dispersion) which occurs because the index of the glass varies slightly depending on the wavelength of the light, and light from real optical transmitters necessarily has nonzero spectral width. Polarization mode dispersion is another source of limitation which occurs because, although the single-mode fiber can sustain only one transverse mode, it can carry this mode with two different polarizations, and slight imperfections or distortions in a 
fiber can alter the propagation velocities for the two polarizations. This phenomenon is called birefringence. [3], [4]

\section{III.DISPERSION COMPENSATION TECHNOLOGIES}

In order to improve overall system performance and reduced as much as possible the transmission performance influenced by the dispersion, several dispersion compensation technologies were proposed. Amongst the various techniques proposed in the literature, the ones that appear to hold immediate promise for dispersion compensation and management could be broadly classified as: dispersion compensating fibers (DCF), chirped fiber bragg gratings ( $\mathrm{FBG})$ and high-order mode (HOM) fiber.

The idea of using dispersion compensation fiber for dispersion compensation was proposed in 1980 but, until after the invention of optical amplifiers, DCF began to be widespread attention and study. As the products of DCF are more mature, stable, not easily affected by temperature, wide bandwidth, DCF has become a most suitable method of dispersion compensation.

There is positive second-order and third-order dispersion value in SMF, while the DCF dispersion value is negative. So by inserting a DCF, the average dispersion is close to zero. [5]

\section{DISPERSION COMPENSATION WITH DCF}

The use of dispersion compensating fibers is an efficient way to upgrade installed links of standard single mode fiber (SMF). Conventional dispersion compensating fibers have a high negative dispersion -70 to $-90 \mathrm{ps} / \mathrm{nm} . \mathrm{km}$ and can be used to compensate the positive dispersion of transmission fiber in C-band.

According to the relative position of DCF and single mode fiber, post-compensation, pre-compensation and symmetrical/mix compensation, is proposed. DCF precompensation scheme achieve dispersion compensation by placing the DCF before a conventional single mode fiber or after the optical transmitter. Post-compensation scheme achieve dispersion compensation by placing the DCF after the single mode fiber. Symmetrical/mix compensation scheme is consist of both pre-compensation and postcompensation. [6]

\section{METHODOLOGY}

We used the Optisystem 7.0 for simulation and designing. It is an innovative, rapid developing and powerful software design tool. It enables the users to test and simulate almost all kinds of optical fiber links.

The Simulation of three dispersion compensation schemes is shown in Fig.1. We use the parameters in Table 1 for the simulation of dispersion compensation systems. In these simulations, the transmitter section consists of data source, modulator driver (NRZ driver), laser source and Mach-Zehnder modulator. Data source produces a pseudorandom sequence of bits at a rate of $10 \mathrm{Gbit} / \mathrm{s}$. The output of data source is given to modulator driver which produces NRZ format pulse with duty cycle of 0.5 . The output of laser source is CW type at frequency $193.1 \mathrm{THz}$ and output power of $5 \mathrm{dBm}$. The modulator is of MachZehnder modulators have the Excitation ratio 30db.The loop control system has two loops. Each span consists of $250 \mathrm{~km}$ of transmission fiber (SMF) and $50 \mathrm{~km} \mathrm{DCF}$ in order to fully compensate for the dispersion slope and accumulated dispersion in the transmission fiber. The total length of fiber channel remains same i.e. $300 \mathrm{~km}$; however it is segmented in the ratio of 1:5 i.e. $50 \mathrm{~km} \mathrm{DCF}$ and 250 $\mathrm{km} \mathrm{SMF}$. Two EDFAs in front of transmission fiber and DCF with $35 \mathrm{~dB}, 25 \mathrm{~dB}$ gain and $4 \mathrm{~dB}$ noise figures each are used to adjust input power levels. At the receiver side, the optical signal is transformed into an electrical signal by a PIN photodiode The PIN photo detector have the responsivity 1A/W and Dark current 10nA. The electrical signal is filtered by a low pass Bessel filter.

Table 1. Simulation parameters

\begin{tabular}{|l|l|}
\hline SMF Parameters & Value \\
\hline Reference wavelength & $1550 \mathrm{~nm}$ \\
\hline Length & $250 \mathrm{~km}$ \\
\hline Attenuation & $0.25 \mathrm{db} / \mathrm{km}$ \\
\hline Dispersion & $17.25 \mathrm{ps} / \mathrm{nm} / \mathrm{km}$ \\
\hline Dispersion slope & $0.08 \mathrm{ps} / \mathrm{nm}^{2} / \mathrm{km}$ \\
\hline PMD coefficient & $0.5 \mathrm{ps} / \mathrm{km}$ \\
\hline Differential group delay & $3 \mathrm{ps} / \mathrm{km}$ \\
\hline & \\
\hline DCF Parameters & Value \\
\hline Reference wavelength & $1550 \mathrm{~nm}$ \\
\hline Length & $50 \mathrm{~km}$ \\
\hline Attenuation & $0.6 \mathrm{db} / \mathrm{km}$ \\
\hline Dispersion & $-80 \mathrm{ps} / \mathrm{nm} / \mathrm{km}$ \\
\hline Dispersion slope & $0.08 \mathrm{ps} / \mathrm{nm}^{2} / \mathrm{km}$ \\
\hline PMD coefficient & $0.5 \mathrm{ps} / \mathrm{km}$ \\
\hline Differential group delay & $3 \mathrm{ps} / \mathrm{km}$ \\
\hline
\end{tabular}

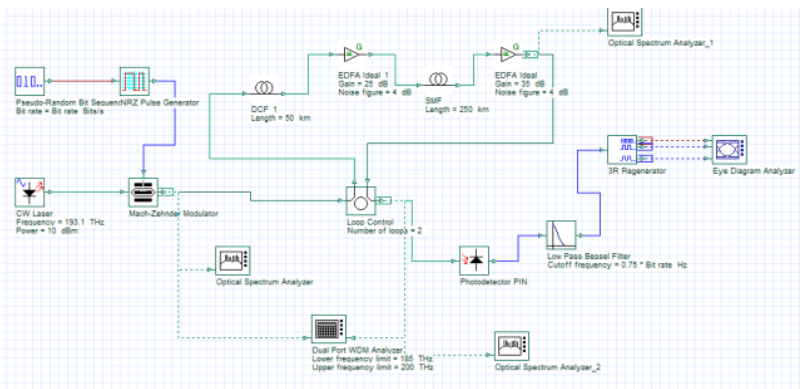

(a) Pre-compensation

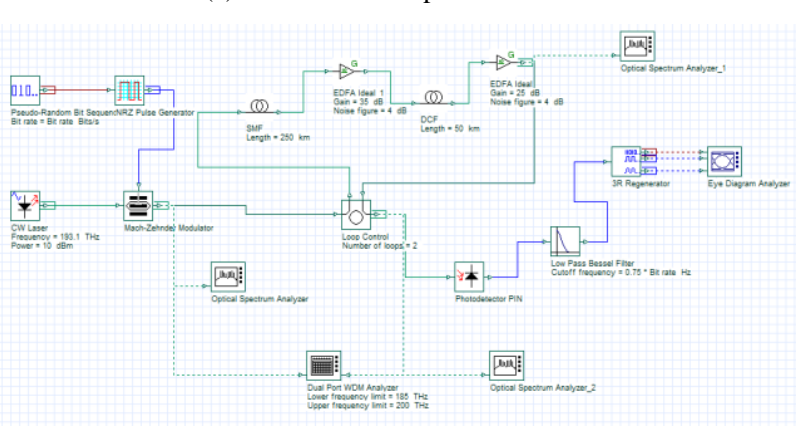

(b) Post-compensation 
International Journal of Advanced Research in Computer and Communication Engineering Vol. 4, Issue 2, February 2015

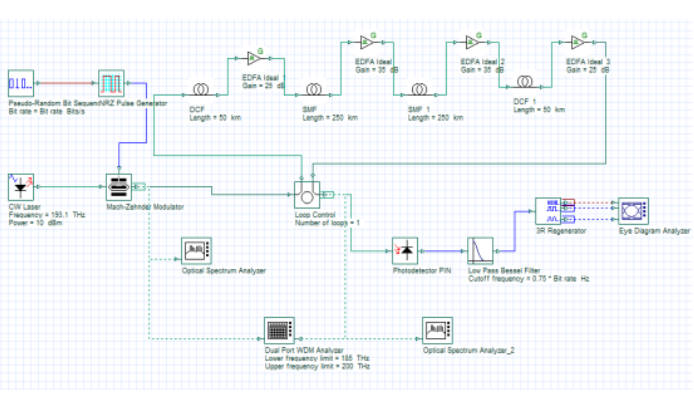

(c) Symmetrical/mix-compensation

Fig. 1: Three dispersion compensation schemes

\section{RESULTS AND ANALYSIS}

The eye diagrams for the three schemes are shown in Fig. 2. The parameters such as Q-factor, Min. BER, Eye height and Threshold value, for the three dispersion compensation schemes are tabulated into table 2 and compared.

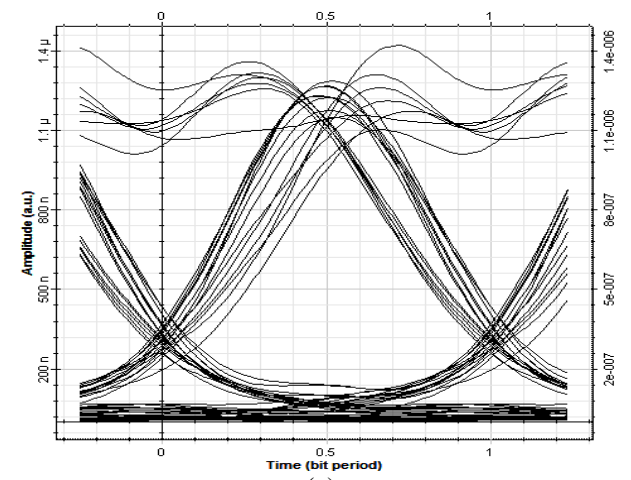

(a)

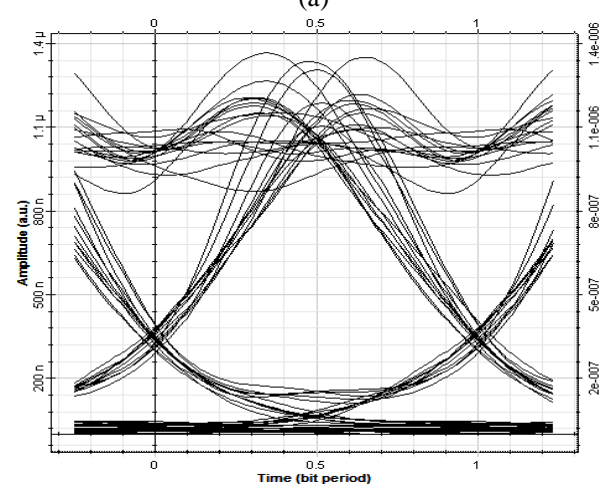

(b)

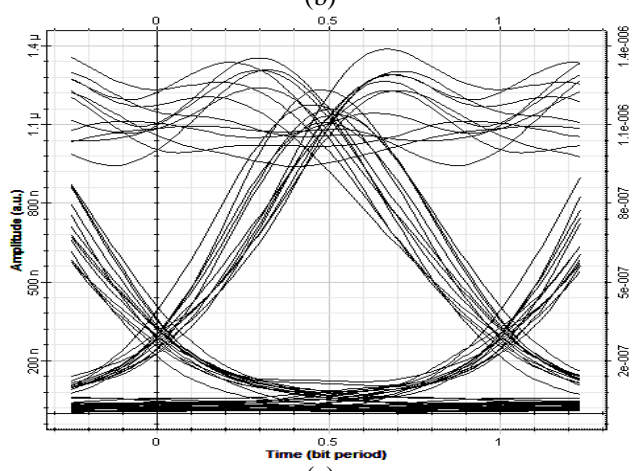

(c)

Fig. 2: Eye diagrams for (a) Pre-compensation, (b) Post-compensation and (c) Symmetrical/mix-compensation.
Table 2. Comparison of three dispersion compensation schemes

\begin{tabular}{|c|c|c|c|}
\hline & $\begin{array}{c}\text { Pre- } \\
\text { compensation }\end{array}$ & $\begin{array}{c}\text { Post- } \\
\text { compensation }\end{array}$ & $\begin{array}{c}\text { Symmetrical- } \\
\text { compensation }\end{array}$ \\
\hline $\begin{array}{c}\text { Q-factor } \\
(\mathrm{db})\end{array}$ & 9.5471 & 7.80473 & 10.4526 \\
\hline BER & $5.12141 \mathrm{e}-022$ & $2.76965 \mathrm{e}-015$ & $5.86482 \mathrm{e}-026$ \\
\hline Eye height & $7.53315 \mathrm{e}-007$ & $6.37824 \mathrm{e}-007$ & $7.48539 \mathrm{e}-007$ \\
\hline $\begin{array}{c}\text { Threshold } \\
\text { value }\end{array}$ & $3.90667 \mathrm{e}-007$ & $3.98982 \mathrm{e}-007$ & $3.67807 \mathrm{e}-007$ \\
\hline
\end{tabular}

From the Table 2 and eye diagrams, we can see that the symmetrical-compensation scheme performs better than the pre-compensation and post-compensation schemes.

\section{CONCLUSION}

We have analyzed dispersion compensation with dispersion compensating fibers (DCF) at $10 \mathrm{Gbits} / \mathrm{sec}$ for $250 \mathrm{~km}$ of SMF and $50 \mathrm{~km}$ of DCF. Three schemes of dispersion compensation (pre-, post-, and symmetrical/mix-compensation) with DCF are proposed in this paper. After analysis, we find that the symmetrical/mix-dispersion compensation scheme is better than pre and post-compensation schemes. To obtain better signal at receiving end, we check for different combinations of SMF length, DCF length and EDFA gain.

\section{REFERENCES}

[1] R.S.Kaler, Ajay K Sharma, and T.S.Kamal, Comparison of pre-, post- and symmetrical- dispersion compensation schemes for $10 \mathrm{~Gb} / \mathrm{s}$ NRZ links using standard and dispersion compensated fibers, Elsevier Optics Communication 209 2002, 107-123.

[2] Bo-Ning HU, Wang Jing, Wang Wei, Rui-Mei Zhao, Analysis on Dispersion Compensation with DCF based on Optisystem, 2nd International Conference on Industrial and Information Systems 2010, 40-43.

[3] S.Shen and A. M. Weiner, Complete Dispersion Compensation for 400-fs Pulse Transmission over 10-km Fiber Link Using Dispersion Compensating Fiber and Spectral Phase Equalizer, IEEE photonics technology letters, vol. 11, no. 7, July 1999, 827-829.

[4] Saurabh Kumar, Prof. A. K. Jaiswal, Er. Mukesh Kumar, Er. Rohini Saxena, Performance Analysis of Dispersion Compensation in Long Haul Optical Fiber with DCF, IOSR Journal of Electronics and Communication Engineering (IOSR-JECE) e-ISSN: 22782834,p- ISSN: 2278-8735, Volume 6, 2013, 19-23.

[5] Ajeet Singh Verma, A. K. Jaiswal, Mukesh Kumar, An Improved Methodology for Dispersion Compensation and Synchronization in Optical Fiber Communication Networks, International Journal of Emerging Technology and Advanced Engineering, ISSN 2250-2459, ISO 9001:2008 Certified Journal, Volume 3, Issue 5, May 201, 769-775.

[6] Gurpreet Kaur, Navdeep Kaur, Use of Dispersion Compensating Fiber in Optical Transmission Network for NRZ Modulation Format, International Journal Of Engineering And Computer Science ISSN: 2319-7242 Volume 3 Issue 5, May 2014, 5839-5842. 